\title{
Correction to: MRI/US fusion-guided biopsy: performing exclusively targeted biopsies for the early detection of prostate cancer
}

\author{
Maurizio Del Monte ${ }^{1} \cdot$ Costantino Leonardo $^{2} \cdot$ Vincenzo Salvo $^{1} \cdot$ Marcello Domenico Grompone $^{1}$. \\ Martina Pecoraro ${ }^{1} \cdot$ Arnaldo Stanzione $^{3} \cdot$ Riccardo Campa $^{1} \cdot$ Francesco Vullo $^{1} \cdot$ Alessandro Sciarra $^{2} \cdot$ Carlo Catalano $^{1}$. \\ Valeria Panebianco ${ }^{1}$
}

Published online: 9 January 2018

(c) Italian Society of Medical Radiology 2018

\section{Correction to: Radiol med \\ https://doi.org/10.1007/s11547-017-0825-8}

In the original publication of the article, the first and last names of the second author were interchanged. The correct name of the author should read as "Costantino Leonardo".

The original article has been updated accordingly.

The original article can be found online at https://doi.org/10.1007/ s11547-017-0825-8.

Valeria Panebianco

valeria.panebianco@gmail.com

1 Prostate Unit-Department of Radiological Sciences, Oncology and Pathology, Sapienza University of Rome, 00161 Rome, Italy

2 Department of Urology, Sapienza University, Rome, Italy

3 Department of Advanced Biomedical Sciences, University "Federico II", Naples, Italy 